\title{
Radiative transfer and Faraday effect in turbulent atmospheres
}

\author{
N. A. Silant'ev ${ }^{1,2}$ \\ ${ }^{1}$ Instituto Nacional de Astrofísica, Óptica y Electrónica, Apartado Postal 51 y 216, Z.P. 72000, Pue. México, Mexico \\ e-mail: silant@inaoep.mx \\ 2 Main Astronomical Observatory of Russian Academy of Sciences, Pulkovo, St.-Petersburg, 196140, Russia
}

Received 23 July 2004 / Accepted 30 November 2004

\begin{abstract}
We investigated the system of transfer equations for the Stokes parameters $I, Q$ and $U$ in turbulent atmospheres. The extinction coefficients $\alpha=\alpha_{0}+\alpha^{\prime}$, the number density of free electrons $n=n_{0}+n^{\prime}$ and magnetic field $\boldsymbol{B}=\boldsymbol{B}_{0}+\boldsymbol{B}^{\prime}$ are considered as stochastic values. The dependence of the averaged Stokes parameters $I_{0}, Q_{0}$ and $U_{0}$ on fluctuating parts of these coefficients is investigated. It is found that the extinction coefficient for the averaged Stokes parameters is smaller than the average value $\alpha_{0}$. The existence of magnetic and number density fluctuations gives rise to an additional decrease of the magnitude of linear polarization. These fluctuations also decrease the positional angle of polarization compared with its mean value. We have found that it is possible to write the radiative transfer equations for $I_{0}, Q_{0}$ and $U_{0}$ with the renormalized coefficients (effective extinction coefficient, Faraday rotation parameter and additional extinction coefficient for polarized radiation components). The only condition for doing this is that the characteristic correlation length $R_{1}$ of turbulence should be small compared to the photon free path. The conservative Milne problem is solved analytically for the case of large Faraday rotation (mean or fluctuating) along the photon free path. The magnetic field fluctuations change drastically the spectrum of linear polarization for large wavelengths. The spectrum acquires the form $\propto 1 / \lambda^{4}$ whereas the atmospheres without magnetic fluctuations give $1 / \lambda^{2}$-dependence. The $\lambda$-dependence of the positional angle $\chi$ also changes considerably.
\end{abstract}

Key words. radiative transfer - turbulence - accretion, accretion disks - polarization - magnetic fields - stars: atmospheres

\section{Introduction}

The theory of radiative transfer in homogeneous magnetized atmospheres has been developed in a number of papers (Basko 1977; Silant'ev 1979, 1982, 1994; Meszaros et al. 1980; Kaminker et al. 1982). For large magnetic fields, $B>$ $10^{5}-10^{6} \mathrm{G}$, when the cross-sections of optical radiation differ from the Thomson value $\sigma_{\mathrm{Th}} \simeq 6.65 \times 10^{-25} \mathrm{~cm}^{2}$, one can neglect the Faraday rotation of elliptically polarized normal modes and consider the system of coupled equations for the intensities of these modes (Nagel 1980; Kaminker et al. 1982). The different intensities of these normal waves give rise to the net polarization of the radiation. For weaker magnetic fields, $B<10^{6} \mathrm{G}$, the normal waves are right and left circular polarized and have Thomson scattering cross-sections. The anisotropy of the magnetized atmosphere in this case is due to the Faraday rotation only. Such atmospheres were considered by Silant'ev (1994), Agol \& Blaes (1996), Agol et al. (1998) and Shternin et al. (2003).

As distinct from the papers mentioned above, we consider here stochastic magnetized atmospheres which we call turbulent. We assume that the turbulence gives rise to fluctuations of the extinction coefficient $\alpha$, number density of free electrons in a plasma $n$, and magnetic field $\boldsymbol{B}$. For simplicity we assume that the characteristic correlation length $R_{1}$ of the turbulent velocity field also characterizes the correlations of all the fluctuating values $-\alpha^{\prime}, n^{\prime}$ and $\boldsymbol{B}^{\prime}$. The turbulence is assumed to be homogeneous and isotropic, i.e. the mean values $\langle\alpha\rangle=\alpha_{0}$, $\langle n\rangle=n_{0}$ and $\langle\boldsymbol{B}\rangle=\boldsymbol{B}_{0}$ are constant. The ensemble averaging is denoted by \langle\rangle .

An ensemble average value such as $\langle I\rangle=I_{0},\langle Q\rangle=Q_{0}$ and $\langle U\rangle=U_{0}$ can be taken as observed value if the time of the observation is greater than the characteristic life time of the turbulent fluctuations, or if the observed volume contains many turbulent "cells". Besides, we restrict ourselves by the supposition that the life time of turbulent correlations is greater than the time of the light propagation over the distance $\sim R_{1}$. So, only simultaneous correlations will appear in our formulas. We consider only the radiation continuum scattering and propagation where one can neglect the Doppler frequency change. In this case the fluctuating values $I^{\prime}, Q^{\prime}$ and $U^{\prime}$ arise as a result of the light propagation in an atmosphere with fluctuating parameters. For this reason we first investigate the process of the light propagation in such a medium. To start, we recall some basic equations.

The angle of the Faraday rotation $\chi$ may be written in the form (Gnedin \& Silant'ev 1997):

$\chi(\boldsymbol{n}, \boldsymbol{B})=\frac{1}{2} \delta \tau_{\mathrm{Th}}$,

$\delta=\frac{3 \lambda}{4 \pi r_{\mathrm{e}}} \cdot \frac{\omega_{B}}{\omega} \cdot \frac{\boldsymbol{n} \cdot \boldsymbol{B}}{B} \simeq 0.8 \lambda^{2}(\mu \mathrm{m}) B(G) \cos \Theta$,

where $\boldsymbol{n}$ is the direction of wave propagation, $\Theta$ is the angle between $\boldsymbol{n}$ and the magnetic field $\boldsymbol{B}, \tau_{\mathrm{Th}}$ is the Thomson optical 
length, $r_{\mathrm{e}}=e^{2} / m c^{2} \approx 2.82 \times 10^{-13} \mathrm{~cm}$ is the classic radius of electron, $\omega_{B}=e B / m c$ is the cyclotron frequency, and $\omega=$ $2 \pi c / \lambda=k c$ is the cyclic frequency of radiation $\left(\omega_{B} / \omega \cong 0.93 \times\right.$ $\left.10^{-8} \lambda(\mu \mathrm{m}) B(G)\right)$.

The photons escape the optically thick atmosphere mainly from the boundary layer with $\tau \approx 1$. If the Faraday rotation angle $\chi$ corresponding to this optical length is about unity, then the outgoing radiation will be depolarized as a result of the summation of radiation fluxes with very different Faraday rotation angles. Only for directions of the light propagation with $\Theta \approx 90^{\circ}$ will the Faraday rotation angle be small, or absent, and depolarization not occur. The diffusion of light in the inner parts of an atmosphere depolarizes the radiation even in the absence of any magnetic field, as a result of multiple photon scattering. For this reason, far from the boundary the radiation is unpolarized. The existence of Faraday rotation only increases the depolarization process. Thus, the polarization of outgoing radiation acquires a peak-like angular dependence with a maximum for $\boldsymbol{n} \perp \boldsymbol{B}$. This means that the polarization terms $Q$ and $U$ make practically no contribution to the process of formation of the angular distribution. These terms also make very little contribution to the formation of the polarization of scattered radiation. So, for large Faraday rotation angles the intensity $I$ obeys the separate transfer equation with the Rayleigh phase function and to find the polarization one should only take into account the contribution of $I$. A detailed discussion of this is given in the papers (Silant'ev 1994, 2002). This idea will be used by the analytical solution of the Milne problem in the end of the paper.

\section{Radiative transfer equations in an atmosphere with Faraday rotation}

We assume that the magnetized atmosphere is characterized by the extinction coefficient $\alpha=n \sigma_{\mathrm{Th}}+k_{\mathrm{a}}$, where $k_{\mathrm{a}}$ is the absorption factor. The degree of absorption is defined as $q=k_{\mathrm{a}} / \alpha$. The total optical depth of the path element $\mathrm{d} s$ is $\mathrm{d} \tau=\alpha \mathrm{d} s$. When $k_{\mathrm{a}}=0$ this is the Thomson optical depth. We consider a plasma with $\omega_{B} / \omega \ll 1$ where only the Faraday rotation is taken into account and the scattering cross-sections have the Thomson value $\sigma_{\mathrm{Th}}$. The radiative transfer equation for the Stokes parameters $I, Q$ and $U$ have the following form (see Silant'ev 1994; Dolginov et al. 1995):

$\frac{\mathrm{d} I(\boldsymbol{n}, s)}{\mathrm{d} s}=-\alpha I(\boldsymbol{n}, s)+n \sigma_{\mathrm{Th}} B_{I}(\boldsymbol{n}, s)+S(s)$,

$\frac{\mathrm{d} Q(\boldsymbol{n}, s)}{\mathrm{d} s}=-\alpha Q(\boldsymbol{n}, s)-n \sigma_{\mathrm{Th}} \delta U(\boldsymbol{n}, s)+n \sigma_{\mathrm{Th}} B_{Q}(\boldsymbol{n}, s)$,

$\frac{\mathrm{d} U(\boldsymbol{n}, s)}{\mathrm{d} s}=-\alpha U(\boldsymbol{n}, s)+n \sigma_{\mathrm{Th}} \delta Q(\boldsymbol{n}, s)+n \sigma_{\mathrm{Th}} B_{U}(\boldsymbol{n}, s)$.

Here $S(s)$ describes possible sources of radiation. The terms $B_{I}, B_{Q}$ and $B_{U}$ describe the contribution of the light scattering by free electrons. Explicit formulae are given for them in e.g. Chandrasekhar (1950). Later we shall give the general formulae for these terms in a very compact form, using the Wigner rotation functions $D_{p q}^{(1)}(\mathrm{Nn})$. The distance $s$ is directed along the light propagation direction $\boldsymbol{n}(\mathrm{d} I / \mathrm{d} s=(\boldsymbol{n} \nabla) I)$. Recall that $Q=I_{x}-I_{y}, U=I_{x^{\prime}}-I_{y^{\prime}}$, where $I_{n}$ is the intensity of light polarized along the $n$-axis; the axes $x^{\prime}$ and $y^{\prime}$ are derived from the $x$ and $y$-axis by a $45^{\circ}$-right-hand rotation. Usually one chooses the $x$-axis to lie in the plane $(n N)$, where $N$ is the unit vector of the outwards normal to the surface of an atmosphere.

Later we shall consider the Milne problem in a semi-infinite atmosphere. To obtain the asymptotic formulae for the case of large Faraday's rotation we shall use the terms $B_{I}, B_{Q}$ and $B_{U}$ depending on the intensity $I$ only. They have the following form:

$$
\begin{aligned}
& B_{I}(\mu, \tau)=\frac{3}{8}\left[\left(1-\mu^{2}\right) S_{0}(\tau)+\left(1+\mu^{2}\right) S_{1}(\tau)\right] \\
& B_{Q}(\mu, \tau)=\frac{3}{8}\left(1-\mu^{2}\right)\left[S_{0}(\tau)-S_{1}(\tau)\right] \\
& B_{U}(\mu, \tau)=0
\end{aligned}
$$

Here $\mu=\boldsymbol{n} \cdot \boldsymbol{N}$ is the cosine of the angle between the direction of light propagation $\boldsymbol{n}$ and the outwards normal to the atmosphere $N$. The term $B_{U}=0$ because we use an $x$-axis lying in the plane $(\boldsymbol{n} N)$. The functions $S_{0}(\tau)$ and $S_{1}(\tau)$ are determined by the integrals:

$S_{0}(\tau)=\int_{-1}^{1} \mathrm{~d} \mu\left(1-\mu^{2}\right) I(\mu, \tau)$,

$S_{1}(\tau)=\frac{1}{2} \int_{-1}^{1} \mathrm{~d} \mu\left(1+\mu^{2}\right) I(\mu, \tau)$.

It is easy to see that the system of Eqs. (3) and (4) can be written as one equation for the auxiliary complex function $R=-Q+i U$ :

$\frac{\mathrm{d} R}{\mathrm{~d} s}=-g R+n \sigma_{\mathrm{Th}} B_{R}$,

where $B_{R}=-B_{Q}+i B_{U}$, and the function $g$ has the form:

$g=\alpha+i n \sigma_{\mathrm{Th}} \delta \equiv g_{0}+g^{\prime}$.

Here we write the mean value $g_{0}$ as

$g_{0}=\alpha_{0}+i n_{0} \sigma_{\mathrm{Th}} \delta_{0}$

and the fluctuating part of $g$

$g^{\prime}=\alpha^{\prime}+i \sigma_{\mathrm{Th}}\left(n^{\prime} \delta_{0}+n_{0} \delta^{\prime}\right)$.

We have omitted in Eq. (13) the small term $n^{\prime} \delta^{\prime}$. Note that the term $\left\langle n^{\prime} \delta^{\prime}\right\rangle=0$ in an isotropic turbulent medium $\left(\left\langle n^{\prime} \delta^{\prime}\right\rangle \propto\right.$ $\left\langle n^{\prime} B_{\|}^{\prime}\right\rangle=0$ because the component $B_{\|}^{\prime}$ of the magnetic field along $\boldsymbol{n}$ can acquire positive and negative values with equal probability). As usuall we denote the mean values by the subscript "zero" and the fluctuations by a prime. 


\section{The propagation of radiation through a layer of a magnetized turbulent medium}

First we consider the propagation of polarized radiation through a layer $s$ of a magnetized turbulent (stochastic) medium. The Stokes parameters of an incident beam of light are $I^{(0)}, Q^{(0)}$ and $U^{(0)}$. The optical depth of the layer $\tau=\tau_{0}+\tau^{\prime}$ is supposed to be smaller than unity so that multiple scattering inside the layer can be neglected. After propagation through the layer with stochastic parameters $\alpha=\alpha_{0}+\alpha^{\prime}$ and $g=g_{0}+g^{\prime}$ the Stokes parameters can be considered as stochastic values:

$$
\begin{aligned}
& I(s)=I_{0}(s)+I^{\prime}(s), \quad Q(s)=Q_{0}(s)+Q^{\prime}(s), \\
& U(s)=U_{0}(s)+U^{\prime}(s) .
\end{aligned}
$$

The exact solutions of Eqs. (2) and (10) without the sources are:

$$
\begin{aligned}
& I(s)=I^{(0)} \mathrm{e}^{-\left(\tau_{0}+\tau^{\prime}\right)}, \\
& R(s)=-Q(s)+i U(s)=R^{(0)} \mathrm{e}^{-\left(\tau_{0}+\tau^{\prime}\right)-2 i\left(\chi_{0}+\chi^{\prime}\right)} .
\end{aligned}
$$

Here we used the relation:

$R(s)=\sqrt{Q^{2}(s)+U^{2}(s)} \mathrm{e}^{-2 i \chi}$,

where the positional angle $\chi=\chi_{0}+\chi^{\prime}$ is determined from the known relation

$\tan 2 \chi=\frac{U}{Q}$

and the polarization magnitude is $P=\sqrt{Q^{2}+U^{2}}$. The degree of polarization is defined as $p=P / I$. It follows from Eq. (16) that

$P(s)=P^{(0)} \mathrm{e}^{-\left(\tau_{0}+\tau^{\prime}\right)}$,

where $P^{(0)}$ is the polarization magnitude of the incident radiation.

The optical depth $\tau$ and the positional angle $\chi$ are equal to:

$\tau=\tau_{0}+\tau^{\prime}=\alpha_{0} s+\int_{0}^{s} \mathrm{~d} s^{\prime} \alpha^{\prime}\left(s^{\prime}\right)$,

$$
\begin{aligned}
\chi(s) & =\chi_{0}+\chi^{\prime} \\
& =\frac{1}{2} n_{0} \sigma_{\mathrm{Th}} \delta_{0} s+\frac{1}{2} \sigma_{\mathrm{Th}} \int_{0}^{s} \mathrm{~d} s^{\prime}\left(n^{\prime}\left(s^{\prime}\right) \delta_{0}+n_{0} \delta^{\prime}\left(s^{\prime}\right)\right) .
\end{aligned}
$$

Remember that we have used the relation $\left\langle n^{\prime} \delta^{\prime}\right\rangle=0$.

Equations (15) and (19) show that the polarization degree does not change its value, $p(s)=p^{(0)}$. This is due to the intensity $I$ and the magnitude of polarization $P$ having the same extinction. The polarization changes only its positional angle.

Earlier we have discussed the conditions where the observed Stokes parameters coincide with the mean values $I_{0}, Q_{0}$ and $U_{0}$. So, we have to average the exact stochastic expressions (15) and (16) to obtain the observable quantities. For this we use a simple Gaussian ensemble of realizations, which very often occurs in nature (see van Kampen 1981; Gardiner 1985). For a Gaussian ensemble the average of the odd number of fluctuating quantities is equal to zero and the average of the even terms is equal to the sum of all possible two-point correlation functions (correlators). Thus, for example,

$$
\begin{aligned}
& \left\langle n^{\prime}(1) n^{\prime}(2) n^{\prime}(3) n^{\prime}(4)\right\rangle=\left\langle n^{\prime}(1) n^{\prime}(2)\right\rangle\left\langle n^{\prime}(3) n^{\prime}(4)\right\rangle \\
& +\left\langle n^{\prime}(1) n^{\prime}(3)\right\rangle\left\langle n^{\prime}(2) n^{\prime}(4)\right\rangle+\left\langle n^{\prime}(1) n^{\prime}(4)\right\rangle\left\langle n^{\prime}(2) n^{\prime}(3)\right\rangle .
\end{aligned}
$$

For brevity, we denote here the coordinates of various points by numbers. It is easy to obtain very useful relations:

$\left\langle\mathrm{e}^{\tau^{\prime}}\right\rangle=\mathrm{e}^{\frac{1}{2}\left\langle\tau^{\prime 2}\right\rangle}$,

$\left\langle a^{\prime} \mathrm{e}^{-\tau^{\prime}}\right\rangle=-\left\langle a^{\prime} \tau^{\prime}\right\rangle \mathrm{e}^{\frac{1}{2}\left\langle\tau^{\prime 2}\right\rangle}$,

where $\tau^{\prime}$ and $a^{\prime}$ are the Gaussian stochastic quantities with $\left\langle\tau^{\prime}\right\rangle=0$ and $\left\langle a^{\prime}\right\rangle=0$.

Using the first relation in Eq. (23) we obtain:

$$
\begin{aligned}
I_{0}(s) & =I^{(0)} \mathrm{e}^{-\left(\tau_{0}-\frac{1}{2}\left\langle\tau^{\prime 2}\right\rangle\right)} \equiv I^{(0)} \mathrm{e}^{-\tau_{\text {eff }}}, \\
R_{0}(s) & =R^{(0)} \mathrm{e}^{-\left(\tau_{0}+2 i \chi_{0}\right)} \mathrm{e}^{\frac{1}{2}\left(\left\langle\tau^{\prime 2}\right\rangle-4\left\langle\chi^{\prime 2}\right\rangle+4 i\left\langle\tau^{\prime} \chi^{\prime}\right\rangle\right)} \\
& \equiv R^{(0)} \mathrm{e}^{-\left(\tau_{\text {eff }}+2\left\langle\chi^{\prime 2}\right\rangle+2 i \chi_{\text {eff }}\right)} .
\end{aligned}
$$

Here we have introduced new notations:

$$
\begin{aligned}
& \tau_{\text {eff }}=\tau_{0}-\frac{1}{2}\left\langle\tau^{\prime 2}\right\rangle, \\
& \chi_{\text {eff }}=\chi_{0}-\left\langle\tau^{\prime} \chi^{\prime}\right\rangle .
\end{aligned}
$$

From Eq. (25) we find:

$P_{0}(s) \equiv \sqrt{Q_{0}^{2}(s)+U_{0}^{2}(s)}=P^{(0)} \mathrm{e}^{-\left(\tau_{\mathrm{eff}}+2\left\langle\chi^{\prime 2}\right\rangle\right)}$.

First of all, we see that the mean quantities are not described by the mean kinetic coefficients; the fluctuations give an additional contribution. The formulae (24)-(25) demonstrate three effects which are due to the stochastic nature of the medium. Let us discuss them in more detail.

1) It is seen that the effective optical depth (26) is smaller than the mean optical depth $\tau_{0}$. This can be understood from very simple model. Consider two realizations of the medium. Let in the first realization the optical depth $\tau_{1}=\tau_{0}+\tau^{\prime}$. Then $I_{1}(s)=I^{(0)} \exp \left(-\tau_{0}-\tau^{\prime}\right)$. In the second realization we take $\tau_{2}=\tau_{0}-\tau^{\prime}$ which gives $I_{2}(s)=I^{(0)} \exp \left(-\tau_{0}+\tau^{\prime}\right)$. The mean value of the optical depth is equal to $\tau_{0}$. The mean intensity is

$$
\begin{aligned}
I_{0}(s) & =\frac{1}{2}\left(I_{1}(s)+I_{2}(s)\right) \\
& =I^{(0)} \exp \left(-\tau_{0}\right) \cosh \left(\tau^{\prime}\right) \geq I^{(0)} \exp \left(-\tau_{0}\right),
\end{aligned}
$$

i.e. the effective optical length is really smaller than $\tau_{0}$. For small $\tau^{\prime}$ we have $\cosh \left(\tau^{\prime}\right) \approx 1+\tau^{\prime 2} / 2 \approx \exp \left(\tau^{\prime 2} / 2\right)$ which coincides with Eq. (24).

2) The second effect describes the decrease of the mean polarization magnitude $P_{0}(s)$ due to fluctuations of the positional angles $\chi$ in various realizations. This effect is rather evident. Actually, the sum of the polarized beams with different positional angles represents a beam that is less polarized than the incident beams. Nevertheless, it is useful to 
demonstrate this using a very simple model from two realizations. Let in the first realization the Faraday rotation angle be $\chi_{1}=\chi_{0}+\chi^{\prime}$, i.e.

$$
\begin{aligned}
& Q_{1}(s)=Q^{(0)} \cos 2\left(\chi_{0}+\chi^{\prime}\right), \\
& U_{1}(s)=Q^{(0)} \sin 2\left(\chi_{0}+\chi^{\prime}\right) .
\end{aligned}
$$

For the second realization we have the same terms but with negative $\chi^{\prime}$. Calculating the mean values $Q_{0}(s)=\left(Q_{1}(s)+\right.$ $\left.Q_{2}(s)\right) / 2$ and $U_{0}(s)=\left(U_{1}(s)+U_{2}(s)\right) / 2$ we obtain

$P_{0}(s)=P^{(0)} \cos 2 \chi^{\prime} \approx P^{(0)}\left(1-2 \chi^{\prime 2}\right) \approx P^{(0)} \mathrm{e}^{-2 \chi^{\prime 2}}$,

which coincides with the corresponding factor in formula (25).

3) The third effect (see Eq. (27)) describes the decrease of positional angle compared with its mean value $\chi_{0}$. The effect does not arise if there is no mean magnetic field $\boldsymbol{B}_{0}$. The physical explanation of this effect is very simple. Let the first realization have an optical depth $\tau_{1}=\tau_{0}+\tau^{\prime}>\tau_{0}$. In this case the Faraday rotation of the polarization plane is greater than for a mean path $\tau_{0}$. But simultaneously the magnitude of polarized component is smaller than for an optical depth $\tau_{0}$. For the second realization with $\tau_{2}=\tau_{0}-\tau^{\prime}$ the Faraday rotation is smaller than that corresponding to $\tau_{0}$, but the magnitude of polarization is greater. As a result, the effective position angle is smaller than the mean value $\chi_{0}$. Without a mean magnetic field this explanation does not work - for every realization there exists with the equal probability magnetic fluctuations $\boldsymbol{B}^{\prime}$ and $-\boldsymbol{B}^{\prime}$ and the net Faraday rotation does not arise.

Now we give the explicit expressions for the mean values $\left\langle\tau^{\prime 2}\right\rangle$, $\left\langle\chi^{\prime 2}\right\rangle$ and $\left\langle\tau^{\prime} \chi^{\prime}\right\rangle$ in Eqs. (24)-(25). According to Eqs. (20) and (21) the values $\tau^{\prime}$ and $\chi^{\prime}$ are represented as the path integrals along the direction of light propagation. We restrict ourselves to the model of homogeneous and isotropic turbulence. In this case the two-point correlation functions depend on the absolute value $R=\left|s^{\prime}-s^{\prime \prime}\right|$. For the derivation of the formulae we use the equality:

$$
\begin{aligned}
\int_{0}^{s} \mathrm{~d} s^{\prime} \int_{0}^{s} \mathrm{~d} s^{\prime \prime} A(R) & =\int_{0}^{s} \mathrm{~d} R A(R) \int_{R}^{2 s-R} \mathrm{~d} r \\
& =2 \int_{0}^{s} \mathrm{~d} R(s-R) A(R) .
\end{aligned}
$$

Here we used the substitution $r=s^{\prime}+s^{\prime \prime}$ and $R=s^{\prime}-s^{\prime \prime}$ and take into account that $A(R)$ is an even function of $R$.

Besides, we introduce the various correlation functions:

$$
\begin{aligned}
& \left\langle\alpha^{\prime}(s) \alpha^{\prime}\left(s^{\prime}\right)\right\rangle=\left\langle\alpha^{\prime 2}\right\rangle A_{\alpha}\left(R / R_{1}\right), \\
& \left\langle n^{\prime}(s) n^{\prime}\left(s^{\prime}\right)\right\rangle=\left\langle n^{\prime 2}\right\rangle A_{n}\left(R / R_{1}\right), \\
& \left\langle\alpha^{\prime}(s) n^{\prime}\left(s^{\prime}\right)\right\rangle=\left\langle\alpha^{\prime} n^{\prime}\right\rangle A_{\alpha n}\left(R / R_{1}\right) .
\end{aligned}
$$

All the $A_{m}$-functions are equal to unity at $R=0$. For an atmosphere without absorption ( $\left.\alpha=n \sigma_{\mathrm{Th}}\right)$ the correlator (32) is proportional to (31).

$$
\left\langle B_{\|}^{\prime}(s) B_{\|}^{\prime}\left(s^{\prime}\right)\right\rangle=\frac{1}{3}\left\langle B^{\prime 2}\right\rangle A_{B}\left(R / R_{1}\right)
$$

Remember that $B_{\|}$is the component of the magnetic field $\boldsymbol{B}$ along the direction of the light propagation. In isotropic turbulence one has $\left\langle B_{\|}^{\prime 2}(s)\right\rangle=(1 / 3)\left\langle B^{\prime 2}\right\rangle$. Because the Faraday rotation parameter $\delta$ (see Eq. (1)) depends linearly on the magnetic field, we have the relation:

$\left\langle\delta^{\prime}(s) \delta^{\prime}\left(s^{\prime}\right)\right\rangle=\left\langle\delta^{\prime 2}\right\rangle A_{B}\left(R / R_{1}\right)$,

where

$\left\langle\delta^{\prime 2}\right\rangle=\frac{1}{3}\left(0.8 \lambda^{2}(\mu \mathrm{m})\right)^{2}\left\langle B^{\prime 2}(G)\right\rangle$.

The values $\left\langle\alpha^{\prime 2}\right\rangle,\left\langle n^{\prime 2}\right\rangle$ and $\left\langle B^{\prime 2}\right\rangle$ characterize the level of fluctuations. In a homogeneous medium they do not depend on position. We assume that the dimensionless correlation functions $A_{\alpha}, A_{n}, A_{\alpha n}$ and $A_{B}$ have the same characteristic correlation length $R_{1} . R_{1}$ is the characteristic length of the turbulence itself. For $R \gg R_{1}$ the correlation practically does not exist. Frequently one takes as a correlation function the expression $\exp \left(-R / R_{1}\right)$.

Introducing the dimensionless variable $x=R / R_{1}$, we obtain the following expressions:

$\left\langle\tau^{\prime 2}\right\rangle=2 \frac{\left\langle\alpha^{\prime 2}\right\rangle}{\alpha_{0}^{2}} \tau_{0} \tau_{1} f_{\alpha}\left(s / R_{1}\right)$.

Here, $\tau_{0}=\alpha_{0} s$ and $\tau_{1}=\alpha_{0} R_{1}$ are the total mean optical depths of the layer and the radius of correlation $R_{1}$, respectively.

$\left\langle\chi^{\prime 2}\right\rangle=\frac{1}{2} \tau_{0}^{(\mathrm{Th})} \tau_{1}^{(\mathrm{Th})}\left[\left\langle\delta^{\prime 2}\right\rangle f_{B}\left(s / R_{1}\right)+\delta_{0}^{2} \frac{\left\langle n^{\prime 2}\right\rangle}{n_{0}^{2}} f_{n}\left(s / R_{1}\right)\right]$,

where $\tau_{0}^{(\mathrm{Th})}=n_{0} \sigma_{\mathrm{Th}} s$ and $\tau_{1}^{(\mathrm{Th})}=n_{0} \sigma_{\mathrm{Th}} R_{1}$ are the mean Thomson optical depths of distances $s$ and $R_{1}$. Note that in Eq. (37) we did not include the third and fourth-order fluctuation terms.

The expression $\left\langle\tau^{\prime} \chi^{\prime}\right\rangle$ has the form:

$\left\langle\tau^{\prime} \chi^{\prime}\right\rangle=2 \tau_{1} \chi_{0} \frac{\left\langle\alpha^{\prime} n^{\prime}\right\rangle}{\alpha_{0} n_{0}} f_{\alpha n}\left(s / R_{1}\right)$

Here the value $\chi_{0}=(1 / 2) n_{0} \sigma_{\mathrm{Th}} s \delta_{0}$ is the mean Faraday rotation angle at the distance $s$.

In all the formulae the functions $f_{m}\left(s / R_{1}\right)$ denote integrals of the type:

$f_{m}\left(s / R_{1}\right)=\int_{0}^{s / R_{1}} \mathrm{~d} x\left(1-x \frac{R_{1}}{s}\right) A_{m}(x)$.

If the distance $s \geq R_{1}$, the functions $f_{m}\left(s / R_{1}\right)$ practically do not depend on the parameter $s / R_{1}$ and can be considered as factors of the order of unity. Further we shall denote these factors as

$f_{m}=\int_{0}^{\infty} \mathrm{d} x A_{m}(x)$

For the correlation function $A_{m}(x)=\exp (-x) f_{m}=1$. 


\section{Transfer equations for average Stokes parameters}

The results of the previous section show that the averaged Stokes parameters $I_{0}, Q_{0}$ and $U_{0}$ depend on the integrals of the fluctuating parameters of the turbulent medium. In general it is impossible to write the usual transfer equations for these values. But for the case of small-scale turbulence this is possible. As we know, the characteristic optical depth in the usual theory of the radiative transfer is equal to unity. If the optical depth of turbulent fluctuations $\tau_{1}=\alpha R_{1} \ll 1$ we can consider that the parameter $s / R_{1} \gg 1$. In this case the expressions $\left\langle\tau^{\prime 2}\right\rangle$, $\left\langle\chi^{\prime 2}\right\rangle$ and $\left\langle\tau^{\prime} \chi^{\prime}\right\rangle$ depend linearly on the distance $s$ which now can be considered a current coordinate.

It is interesting to note that the expressions (36)-(38) for the $s \gg R_{1}$ - limit can be obtained without the supposition that the ensemble of realizations is Gaussian. They follow also from the nonlinear theory of turbulent diffusion (Silant'ev 2004) if this theory is applied to our initial stochastic Eqs. (2)-(4). This theory does not use the supposition that the ensemble is Gaussian; instead it takes into account the infinite series of twopoint correlation functions.

Using Eqs. (24), (26) and (36), we derive the following equation for the average intensity $I_{0}(\boldsymbol{n}, \boldsymbol{r})$ :

$(\boldsymbol{n} \nabla) I_{0}(\boldsymbol{n}, \boldsymbol{r})=-\alpha_{\mathrm{eff}} I_{0}(\boldsymbol{n}, \boldsymbol{r})+\left\langle n \sigma_{\mathrm{Th}} B_{I}(\boldsymbol{n}, \boldsymbol{r})\right\rangle+\langle S(\boldsymbol{r})\rangle$,

where the effective extinction coefficient is equal to:

$\alpha_{\text {eff }}=\alpha_{0}\left(1-\frac{\left\langle\alpha^{2}\right\rangle}{\alpha_{0}^{2}} \tau_{1} f_{\alpha}\right)$.

Using the second relation of Eq. (23) we can obtain the term $\alpha_{\mathrm{eff}} I_{0}$ in a more formal manner:

$\alpha_{\text {eff }} I_{0}=\langle\alpha I\rangle=\alpha_{0} I_{0}+\left\langle\alpha^{\prime} I^{\prime}\right\rangle \propto\left\langle\alpha \mathrm{e}^{-\int_{0}^{s} \mathrm{~d} s^{\prime} \alpha\left(s^{\prime}\right)}\right\rangle$.

This means that for $s \gg R_{1}$ we have $\left\langle\alpha^{\prime} I^{\prime}\right\rangle=-I_{0}\left\langle\alpha^{2}\right\rangle \tau_{1} f_{\alpha} / \alpha_{0}$. This formal procedure will be used below in the derivation of integral scattering terms in transfer equations for the averaged Stokes parameters $I_{0}, Q_{0}$ and $U_{0}$.

To obtain the term $\left\langle n \sigma_{\mathrm{Th}} B_{I}(\boldsymbol{n}, \boldsymbol{r})\right\rangle$ we can use the second formula in Eq. (23). But first we obtain this term for the case of a conservative atmosphere using a more straightforward approach. To obtain this term we have to know the expression:

$\left\langle n \sigma_{\mathrm{Th}} I(s)\right\rangle \propto\left\langle n \sigma_{\mathrm{Th}} \mathrm{e}^{-\int_{0}^{s} \mathrm{~d} s^{\prime} n \sigma_{\mathrm{Th}}}\right\rangle \simeq-\frac{\mathrm{d}}{\mathrm{d} s}\left\langle\mathrm{e}^{-\alpha_{\mathrm{eff}}^{(\mathrm{Th})} s}\right\rangle \propto \alpha_{\mathrm{eff}}^{(\mathrm{Th})} I_{0}$,

where $\alpha_{\mathrm{eff}}^{(\mathrm{Th})}$ corresponds to the Thomson scattering:

$\alpha_{\mathrm{eff}}^{(\mathrm{Th})}=n_{0} \sigma_{\mathrm{Th}}\left(1-\frac{\left\langle n^{\prime 2}\right\rangle}{n_{0}^{2}} \tau_{1}^{(\mathrm{Th})} f_{n}\right)$.

Of course, expression (43) can also be obtained from Eq. (23). As a result, Eq. (41) for the conservative atmosphere acquires the form:

$(\boldsymbol{n} \nabla) I_{0}(\boldsymbol{n}, \boldsymbol{r})=-\alpha_{\mathrm{eff}}^{(\mathrm{Th})} I_{0}(\boldsymbol{n}, \boldsymbol{r})+\alpha_{\mathrm{eff}}^{(\mathrm{Th})} B_{I}^{\mathrm{eff}}(\boldsymbol{n}, \boldsymbol{r})$,

where $B_{I}^{\text {eff }}$ corresponds to Eq. (5) with $I_{0}$ instead of $I$. Thus, for a conservative atmosphere equation for the mean intensity
$I_{0}$ can be obtained from the usual transfer Eq. (2) by substituting $\alpha_{\mathrm{eff}}^{(\mathrm{Th})}$ for the extinction coefficient $\alpha$ and $I_{0}$ for $I$.

In the conservative case the exact Eq. (2) gives rise to the law of conservation of the radiative flux:

$\frac{\mathrm{d} F(\tau)}{\mathrm{d} \tau}=0, \quad F=2 \pi \int_{-1}^{1} \mathrm{~d} \mu \mu I(\mu, \tau)$.

The averaging of Eq. (46) gives the flux conservation law for an average flux $\langle F\rangle=F_{0}$. Equation (45) also confirms this law.

For an absorbing atmosphere, using the second formula in Eq. (23), we obtain:

$(\boldsymbol{n} \nabla) I_{0}(\boldsymbol{n}, \boldsymbol{r})=-\alpha_{\mathrm{eff}} I_{0}(\boldsymbol{n}, \boldsymbol{r})+\beta_{\mathrm{eff}} B_{I}^{\mathrm{eff}}(\boldsymbol{n}, \boldsymbol{r})$,

where the coefficient $\beta_{\text {eff }}$ is equal to:

$\beta_{\text {eff }}=n_{0} \sigma_{\mathrm{Th}}\left[1-\tau_{1} \frac{\left\langle\alpha^{\prime} n^{\prime}\right\rangle}{\alpha_{0} n_{0}} f_{\alpha n}\right]$.

Remember that $\tau_{1}=\alpha_{0} R_{1}$ is the total optical depth of a fluctuation region. The coefficients $\alpha_{\text {eff }}$ and $\beta_{\text {eff }}$ coincide only for the conservative atmosphere.

The equation for $R_{0}(\boldsymbol{n}, \boldsymbol{r})=-Q_{0}+i U_{0}$ for a turbulent atmosphere acquires the form:

$(\boldsymbol{n} \nabla) R_{0}(\boldsymbol{n}, \boldsymbol{r})=-g_{\mathrm{eff}} R_{0}(\boldsymbol{n}, \boldsymbol{r})+\left\langle n \sigma_{\mathrm{Th}} B_{R}(\boldsymbol{n}, \boldsymbol{r})\right\rangle$,

where the renormalized coefficient $g_{\text {eff }}$ is:

$g_{\mathrm{eff}}(\boldsymbol{n}) \equiv g_{\mathrm{eff}}^{(\alpha)}+i g_{\mathrm{eff}}^{(\chi)}$,

$g_{\mathrm{eff}}^{(\alpha)}=\alpha_{\mathrm{eff}}+n_{0} \sigma_{\mathrm{Th}} \tau_{1}^{(\mathrm{Th})}\left[\left\langle\delta^{\prime 2}\right\rangle f_{B}+\delta_{0}^{2} \frac{\left\langle n^{\prime 2}\right\rangle}{n_{0}^{2}} f_{n}\right]$,

$g_{\mathrm{eff}}^{(\chi)}=n_{0} \sigma_{\mathrm{Th}} \delta_{0}\left[1-2 \tau_{1} \frac{\left\langle\alpha^{\prime} n^{\prime}\right\rangle}{\alpha_{0} n_{0}} f_{\alpha n}\right]$.

Note that the parameter $\delta_{0}$ depends on the direction of the light propagation $\boldsymbol{n}$ and the value $\left\langle\delta^{\prime 2}\right\rangle \propto\left\langle B^{\prime 2}\right\rangle$ does not depend on the angles because of the assumed isotropy of the turbulence.

The problem of evaluating the term $\left\langle n \sigma_{\mathrm{Th}} B_{R}\right\rangle$ is the same as in the previous case because in this section we restrict ourselves to the approximate expressions for $B_{I}, B_{Q}$ and $B_{U}$ which depend only on the intensity $I$. In the next section we shall present the solution for the general case where $B_{I}$ and $B_{R}$ depend on all the Stokes parameters. Here we give the renormalized equation for $R_{0}$ when $B_{Q}$ and $B_{U}$ depend on the intensity $I$ only (see Eqs. (6)-(9)). For the conservative case we have:

$(\boldsymbol{n} \nabla) Q_{0}(\boldsymbol{n}, \boldsymbol{r})=-g_{\mathrm{Th}}^{(\alpha)} Q_{0}(\boldsymbol{n}, \boldsymbol{r})-g_{\mathrm{Th}}^{(\chi)} U_{0}(\boldsymbol{n}, \boldsymbol{r})+\alpha_{\mathrm{eff}}^{(\mathrm{Th})} B_{Q}^{(\mathrm{eff})}(\boldsymbol{n}, \boldsymbol{r})$,

$(\boldsymbol{n} \nabla) U_{0}(\boldsymbol{n}, \boldsymbol{r})=-g_{\mathrm{Th}}^{(\alpha)} U_{0}(\boldsymbol{n}, \boldsymbol{r})+g_{\mathrm{Th}}^{(\chi)} Q_{0}(\boldsymbol{n}, \boldsymbol{r})+\alpha_{\mathrm{eff}}^{(\mathrm{Th})} B_{U}^{(\mathrm{eff})}(\boldsymbol{n}, \boldsymbol{r})$.

The value $\alpha_{\mathrm{eff}}^{(\mathrm{Th})}$ is given in Eq. (44). The $g_{\mathrm{Th}}^{(\alpha)}$-coefficient coincides with Eq. (51) where instead of $\alpha_{\text {eff }}$ one has to take the $\alpha_{\mathrm{eff}}^{(\mathrm{Th})}$-value. The coefficient $g_{\mathrm{Th}}^{(\chi)}$ is:

$g_{\mathrm{Th}}^{(\chi)}=n_{0} \sigma_{\mathrm{Th}} \delta_{0}\left[1-2 \tau_{1}^{(\mathrm{Th})} \frac{\left\langle n^{\prime 2}\right\rangle}{n_{0}^{2}} f_{n}\right]$.

The terms $B_{Q}^{\mathrm{eff}}$ and $B_{U}^{\mathrm{eff}}$ coincide with Eqs. (6) and (7) where if one takes $I_{0}$ instead of $I$. For absorbing atmosphere one has to take the value $\beta_{\text {eff }}$ instead of $\alpha_{\text {eff }}^{(\mathrm{Th})}$ and expression (51) with $\alpha_{\text {eff }}$. 


\section{Transfer equation for the radiation density matrix}

To take into account the contribution of the parameters $Q$ and $U$ in the scattering terms $B_{I}, B_{Q}$ and $B_{U}$ we use the density matrix representation of the radiation. Because for $\omega_{B} / \omega \ll 1$ the circularly polarized waves have independent refractive indices we use the cyclic unit vectors $\boldsymbol{e}_{-1}=\left(\boldsymbol{e}_{x}+i \boldsymbol{e}_{y}\right) / \sqrt{2}, \boldsymbol{e}_{0}=$ $\boldsymbol{e}_{z}, \boldsymbol{e}_{1}=-\left(\boldsymbol{e}_{x}-i \boldsymbol{e}_{y}\right) / \sqrt{2}$. In the cyclic coordinates the density matrix $\rho_{\alpha \beta}(\boldsymbol{n}, \boldsymbol{r})$ has the form:

$\rho_{-1-1}=(I+V) / 2, \quad \rho_{11}=(I-V) / 2$,

$\rho_{-11}=(-Q+i U) / 2, \quad \rho_{1-1}=(-Q-i U) / 2$.

Here, $V$ is the fourth Stokes parameter which is equal to the intensity of circularly polarized radiation. This parameter obeys a separate transfer equation which we do not consider in this paper.

According to Silant'ev $(1982,1994)$ and Dolginov et al. (1995) the transfer equation for $\rho_{\alpha \beta}$ has the following form:

$$
\begin{aligned}
(\boldsymbol{n} \nabla) \rho_{\alpha \beta}(\boldsymbol{n}, \boldsymbol{r})= & -g_{\alpha \beta}(\boldsymbol{n}) \rho_{\alpha \beta}(\boldsymbol{n}, \boldsymbol{r}) \\
& +\frac{3}{8 \pi} n \sigma_{\mathrm{Th}} a_{p q}^{\alpha \beta}(\boldsymbol{n}) S_{p q}(\boldsymbol{r})+\frac{1}{2} \delta_{\alpha \beta} k_{\mathrm{a}} B_{\omega}(T),
\end{aligned}
$$

where $k_{\mathrm{a}}$ is the absorption factor and $B_{\omega}(T)$ is the Planck function,

$a_{p q}^{\alpha \beta}(\boldsymbol{n})=D_{p \alpha}^{(1)}(\boldsymbol{N n}) D_{q \beta}^{(1) *}(\boldsymbol{N n})$,

$S_{p q}(\boldsymbol{r})=\int \mathrm{d} \boldsymbol{n}^{\prime} a_{p q}^{\gamma v *}\left(\boldsymbol{n}^{\prime}\right) \rho_{\gamma v}\left(\boldsymbol{n}^{\prime}, \boldsymbol{r}\right)$.

The matrix $g_{\alpha \beta}(\boldsymbol{n})$ has the components:

$g_{-1-1}=g_{11}=\alpha$,

$g_{-11}=g_{1-1}^{*}=\alpha+i n \sigma_{\mathrm{Th}} \delta$.

The Wigner functions $D_{p q}^{(1)}(\boldsymbol{N n}) \equiv \boldsymbol{e}_{p}^{(N)} \cdot \boldsymbol{e}_{q}^{(n) *}$ describe the rotation from a coordinate system $(N)$ with $z$-axis along $N$ to a system (n) with $z$-axis along $\boldsymbol{n} ; \boldsymbol{e}_{p}^{(N)}$ and $\boldsymbol{e}_{q}^{(n)}$ are the corresponding cyclic unit vectors of these systems. The functions $D_{p q}^{(1)}(\mathbf{N n})$ are orthogonal and obey the group property (see Varshalovich et al. 1988):

$D_{p q}^{(1)}(\boldsymbol{N n})=D_{p s}^{(1)}(\boldsymbol{N M}) D_{s q}^{(1)}(\boldsymbol{M n})$.

Note that in all the formulae except the first term in the righthand part of Eq. (57), we assume summation over repeated indices. The Wigner functions depend on the Euler angles of rotation:

$D_{p q}^{(1)}(\boldsymbol{N n})=\mathrm{e}^{-i p \varphi} d_{p q}^{(1)}(\vartheta) \mathrm{e}^{-i q \gamma}$

where

$d_{-1-1}^{(1)}=d_{11}^{(1)}=(1+\cos \vartheta) / 2, \quad d_{00}^{(1)}=\cos \vartheta$,

$d_{-11}^{(1)}=d_{1-1}^{(1)}=(1-\cos \vartheta) / 2$,

$d_{01}^{(1)}=d_{-10}^{(1)}=-d_{10}^{(1)}=-d_{0-1}^{(1)}=\sin \vartheta / \sqrt{2}$.
The angles $\varphi$ and $\vartheta$ are the usual azimuthal and polar angles of vector $\boldsymbol{n}$ in the system $(\boldsymbol{N})$. The $\gamma$-angle describes the rotation of the $x_{n}$ and $y_{n}$ axes around the direction $\boldsymbol{n}$. Usually one takes $\gamma=0$. This means that the $x_{n}$-axis lies in the plane $(\boldsymbol{n N})$.

Using the second formula in Eq. (23) to derive the value $\left\langle n \rho_{\gamma v}\right\rangle$ in Eq. (59), we obtain the transfer equation:

$$
\begin{aligned}
(\boldsymbol{n} \nabla)\left\langle\rho_{\alpha \beta}(\boldsymbol{n}, \boldsymbol{r})\right\rangle= & -g_{\alpha \beta}^{\mathrm{eff}}(\boldsymbol{n})\left\langle\rho_{\alpha \beta}(\boldsymbol{n}, \boldsymbol{r})\right\rangle \\
& +\frac{3}{8 \pi} n_{0} \sigma_{\mathrm{Th}} a_{p q}^{\alpha \beta}(\boldsymbol{n}) S_{p q}^{\mathrm{eff}}(\boldsymbol{r})+\frac{1}{2} \delta_{\alpha \beta}\left\langle k_{\mathrm{a}} B_{\omega}(T)\right\rangle .
\end{aligned}
$$

Here the components of $g_{\alpha \beta}^{\text {eff }}(\boldsymbol{n})$ are:

$g_{-1-1}^{\mathrm{eff}}=g_{11}^{\mathrm{eff}}=\alpha_{\mathrm{eff}}$,

$g_{-11}^{\mathrm{eff}}=g_{1-1}^{\mathrm{eff} *}=g_{\mathrm{eff}}^{(\alpha)}+i g_{\mathrm{eff}}^{(\chi)}$.

The coefficients $\alpha_{\mathrm{eff}}, g_{\mathrm{eff}}^{(\alpha)}$ and $g_{\mathrm{eff}}^{(\chi)}$ are presented in Eqs. (42), (51) and (52), respectively. The term $S_{p q}^{\text {eff }}$ is equal to:

$S_{p q}^{\mathrm{eff}}(\boldsymbol{r})=\int \mathrm{d} \boldsymbol{n}^{\prime} a_{p q}^{\gamma v *}\left(\boldsymbol{n}^{\prime}\right) h_{\gamma v}\left\langle\rho_{\gamma v}\left(\boldsymbol{n}^{\prime}, \boldsymbol{r}\right)\right\rangle$.

where the matrix $h_{\gamma v}$ has the components:

$h_{-1-1}=h_{11}=\frac{\beta_{\mathrm{eff}}}{n_{0} \sigma_{\mathrm{Th}}}$,

$h_{-11}=h_{1-1}^{*}=1-\tau_{1} \frac{\left\langle\alpha^{\prime} n^{\prime}\right\rangle}{\alpha_{0} n_{0}} f_{\alpha n}-i \delta_{0} \tau_{1}^{(\mathrm{Th})} \frac{\left\langle n^{\prime 2}\right\rangle}{n_{0}^{2}} f_{n}$.

Note that $h_{-11}=h_{-11}\left(\boldsymbol{n}^{\prime}\right)$ because the value $\delta_{0}$ depends on $\boldsymbol{n}^{\prime}$.

In the next section we will deal with the Milne problem in a semi-infinite conservative (non-absorbing) atmosphere where all the formulae acquire a simpler form. Remember that in Milne's problem the sources of radiation are at infinity. It is convenient to introduce an effective optical depth $\mathrm{d} \tau_{\text {eff }}=\alpha_{\text {eff }}^{(\mathrm{Th})} \mathrm{d} z$ with the $z$-axis directed inside the atmosphere. The transfer equation for $\left\langle\rho_{\alpha \beta}\left(\boldsymbol{n}, \tau_{\mathrm{eff}}\right)\right\rangle$ acquires the form:

$$
\begin{aligned}
\mu \frac{\mathrm{d}}{\mathrm{d} \tau_{\mathrm{eff}}}\left\langle\rho_{\alpha \beta}\left(\boldsymbol{n}, \tau_{\mathrm{eff}}\right)\right\rangle= & \bar{g}_{\alpha \beta}^{\mathrm{eff}}(\boldsymbol{n})\left\langle\rho_{\alpha \beta}\left(\boldsymbol{n}, \tau_{\mathrm{eff}}\right)\right\rangle \\
& -\frac{3}{8 \pi} a_{p q}^{\alpha \beta}(\boldsymbol{n}) S_{p q}^{\mathrm{eff}}\left(\tau_{\mathrm{eff}}\right) .
\end{aligned}
$$

The dimensionless matrix $\bar{g}_{\alpha \beta}^{\mathrm{eff}}$ has the following components:

$\bar{g}_{-1-1}^{\mathrm{eff}}=\bar{g}_{11}^{\mathrm{eff}}=1$,

$\bar{g}_{-11}^{\mathrm{eff}}=\bar{g}_{1-1}^{\mathrm{eff} *}=1+\frac{b}{1-a}+i \delta_{0} \frac{1-2 a}{1-a}$.

Here, we introduce, for brevity, two new values:

$a=\tau_{1}^{(\mathrm{Th})} \frac{\left\langle n^{\prime 2}\right\rangle}{n_{0}^{2}} f_{n}$,

and

$b=\tau_{1}^{(\mathrm{Th})}\left[\left\langle\delta^{\prime 2}\right\rangle f_{B}+\delta_{0}^{2} \frac{\left\langle n^{\prime 2}\right\rangle}{n_{0}^{2}} f_{n}\right]$. 
In the conservative case the matrix $h_{\alpha \beta}$ in Eq. (67) has the very simple form:

$h_{-1-1}=h_{11}=1$,

$h_{-11}=h_{1-1}^{*}=1-i \delta_{0} \frac{a}{1-a}$.

For the conservative case our transfer Eq. (67) for $\boldsymbol{B}=0$ formally coincides with the initial Eq. (57) if there one uses the optical depth notation $\mathrm{d} \tau=n_{0} \sigma_{\mathrm{Th}} \mathrm{d} z$. For an atmosphere with absorption these equations differ in the term $S_{p q}$. As we have seen, the same occurs for separate equations for the intensity only.

The transfer Eqs. (63) and (67) in small-scale turbulent atmospheres can be solved by the same technique as usual transfer equations.

\section{The Milne problem in turbulent atmosphere with Faraday rotation}

The Milne problem describes the radiative transfer in a semi-infinite conservative plane-parallel atmosphere when the sources of radiation are located very far from the surface. The radiative flux (see Eq. (46)) is assumed to have a constant value at all distances from the surface. Ambartsumyan (1942) and Chandrasekhar (1950) found the solution of the Milne problem using the invariance principle. This principle states that all characteristics of outgoing radiation are the same if a layer is added to (or substracted from) a semi-infinite atmosphere. In Silant'ev (1982, 1994) and Dolginov et al. (1995) this principle was used to solve a number of standard problems (the Milne problem, atmospheres with the sources $\propto \tau^{n}, \exp (-h \tau)$ for atmospheres with homogeneous magnetic field $\boldsymbol{B}_{0}$.

In Silant'ev (2002) the exact solutions (Silant'ev 1994) were used to obtain the asymptotic formulae $\left(\delta_{0} \gg 1\right)$ for the standard problems mentioned above. These very simple formulae have the major advantage of giving an analytic description of the polarization for an arbitrary arrangement of the magnetic field in the atmosphere. As far as the Milne problem is concerned, comparison with the exact numerical solution for a magnetic field perpendicular to the surface (Shernin et al. 2003) demonstrates a good agreement of the asymptotic formula with the numerical solution. Thus, for $\delta_{0}=10$ the relative error is $\simeq 10 \%$.

Here we generalize this asymptotic formula for the case of the Milne problem in turbulent magnetized atmosphere. As it was discussed in the introduction, in a magnetized atmosphere the polarization terms $Q$ and $U$ make a very small contribution to the process of the formation of angular distribution and polarization. As a result, the intensity $I$ obeys a separate transfer equation with the Rayleigh phase function:

$$
\begin{aligned}
(\boldsymbol{n} \nabla) I(\boldsymbol{n}, \boldsymbol{r})= & -\alpha I(\boldsymbol{n}, \boldsymbol{r})+\frac{3}{16 \pi} n \sigma_{\mathrm{Th}} \\
& \times \int \mathrm{d} \boldsymbol{n}^{\prime}\left[1+\left(\boldsymbol{n} \cdot \boldsymbol{n}^{\prime}\right)^{2}\right] I(\boldsymbol{n}, \boldsymbol{r})+S(\boldsymbol{r}) .
\end{aligned}
$$

\subsection{Derivation of the asymptotic formula for non-turbulent atmospheres}

For a plane-parallel atmosphere Eq. (72) gives rise to Eqs. (2) and (5). From these equations it is easy to derive the integral equation for $S_{0}(\boldsymbol{r})$ and $S_{1}(\boldsymbol{r})$.

It is known (Chandrasekhar 1950) that the Milne problem for Eq. (72) gives the following expression for the intensity of outgoing radiation:

$I(\mu, 0)=\frac{F}{2 \pi H_{1}} H(\mu)$

where the $H$-function obeys the nonlinear equation:

$H(\mu)=1+\mu H(\mu) \int_{0}^{1} \mathrm{~d} \mu^{\prime} \frac{\Psi\left(\mu^{\prime}\right) H\left(\mu^{\prime}\right)}{\mu+\mu^{\prime}}$.

Here,

$\Psi(\mu)=\frac{3}{16}\left(3-\mu^{2}\right)$

$H_{n}=\int_{0}^{1} \mathrm{~d} \mu \mu^{n} H(\mu)$

Chandrasekhar (1950) gives $H_{0}=2.06088, H_{1}=1.19400$, $H_{2}=0.84940$. Remember that $H(\mu)$ grows monotonically from 1 at $\mu=0$ up to 3.02 at $\mu=1$.

The outgoing radiation can be represented by the integrals:

$I(\mu, 0)=\int_{0}^{\infty} \frac{\mathrm{d} \tau}{\mu} \exp \left[-\frac{\tau}{\mu}\right] B_{I}(\mu, \tau)$,

$R(\mu, 0)=\int_{0}^{\infty} \frac{\mathrm{d} \tau}{\mu} \exp \left[-\left(1+i \delta_{0}\right) \frac{\tau}{\mu}\right] B_{R}(\mu, \tau)$.

Remember that $R(\mu, \tau)=-Q(\mu, \tau)+i U(\mu, \tau)$ and $B_{R}=-B_{Q}+$ $i B_{U}$.

For large Faraday rotations $\left(\delta_{0} \gg 1\right)$ one can take $B_{R}(\mu, \tau)$ at $\tau=0$. This gives the asymptotic formula:

$R(\mu, 0) \simeq-\frac{3}{8}\left(1-\mu^{2}\right) \frac{S_{0}(0)-S_{1}(0)}{1+i \delta_{0}}$.

The general theory (see Silant'ev 1994) gives a system of linear equations to find the values $S_{0}(0)$ and $S_{1}(0)$. The coefficients of this system are combinations of $H_{n}$. As a result, one can obtain:

$R(\mu, 0)=0.09138 \frac{F}{2 \pi H_{1}} \cdot \frac{1-\mu^{2}}{1+i \delta_{0}}$.

Remember that $\delta_{0}=0.8 \lambda^{2}(\mu m) B_{0}(G) \cos \Theta, \cos \Theta=$ $\boldsymbol{n} \cdot \boldsymbol{B}_{0} / B_{0}, \mu=\cos \vartheta=\boldsymbol{n} \cdot \boldsymbol{N}, \boldsymbol{N}$ is the outwards normal to the atmosphere.

Expression (79) gives for the degree of polarization:

$p(\boldsymbol{n})=-9.14 \% \frac{\left(1-\mu^{2}\right)}{H(\mu)} \cdot \frac{1}{\sqrt{1+\delta_{0}^{2}}}$.

The minus sign denotes that the plane of polarization is perpendicular to the plane $(\boldsymbol{n N})$. For the positional angle $\chi$ formula (79) gives:

$\tan 2 \chi=\delta_{0}$. 
For a magnetic field $\boldsymbol{B}_{0} \| \mid \boldsymbol{N}$ we have $p_{\max }(0)=9.14 \%$ instead of the known Chandrasekhar-Sobolev value $11.71 \%$. The exact numerical calculations (Shternin et al. 2003) confirm this result.

\subsection{Derivation of the asymptotic formula for turbulent atmospheres}

Now we consider the Milne problem in a turbulent atmosphere. As was mentioned, Eq. (67) formally coincides with Eq. (57). The difference (remember that all quantities depend on intensity $I$ only!) between them is that the term $\left(1+i \delta_{0}\right)$ has been replaced by the term

$\left(1+\frac{b}{1-a}+i \delta_{0} \frac{1-2 a}{1-a}\right)$

The difference in the definition of optical depths $-\mathrm{d} \tau=$ $n_{0} \sigma_{\mathrm{Th}} \mathrm{d} z$ for the usual Eq. (57), and $\mathrm{d} \tau_{\mathrm{eff}}=\alpha_{\mathrm{eff}}^{(\mathrm{Th})} \mathrm{d} z$ for Eq. (67) gives rise to the relation: $S_{p}^{\text {eff }}\left(\tau_{\text {eff }}\right)=S_{p}((1-a) \tau)$. This means that at $\tau=0$ these values coincide. Thus, the value $R(\boldsymbol{n}, 0)$ is expressed by the same formula (77) but with Eq. (82) instead of $\left(1+i \delta_{0}\right)$. This gives the asymptotic expression for the turbulent medium:

$R(\boldsymbol{n}, 0)=0.09138 \frac{F}{2 \pi H_{1}} \cdot \frac{1-\mu^{2}}{1+\frac{b}{1-a}+i \delta_{0} \frac{1-2 a}{1-a}}$.

This expression gives the other formulae for the polarization degree $p=P / I$ and the positional angle $\chi$ :

$p(\boldsymbol{n})=-9.14 \% \frac{\left(1-\mu^{2}\right)}{H(\mu)} \cdot \frac{1}{\sqrt{\left(1+\frac{b}{1-a}\right)^{2}+\delta_{0}^{2}\left(\frac{1-2 a}{1-a}\right)^{2}}}$,

$\tan 2 \chi=\frac{\delta_{0}(1-2 a)}{1-a+b}$

It follows from Eq. (70) that $b \propto \lambda^{4}$ whereas $\delta_{0} \propto \lambda^{2}$. For this reason, the polarization spectrum for large wavelengths is $\propto 1 / \lambda^{4}$ in contrast to Eq. (80) which gives $p \propto 1 / \lambda^{2}$. This difference in spectrum can be used to confirm the existence of the turbulence in an atmosphere.

Formula (81) demonstrates that for large wavelengths $\left(\delta_{0} \gg 1\right)$ the positional angle $\chi$ tends to a value $45^{\circ}$ as compared with the plane $(\boldsymbol{n} \boldsymbol{N})$. The behaviour of $\chi$ in a turbulent atmosphere differs strongly from that in the non-turbulent case. Only for small $\lambda$ both Eqs. (81) and (85) give $\chi \propto \lambda^{2}$. In the turbulent case the angle $\chi$ acquires a maximum value at $\lambda_{*}$ which can be found from the relation:

$\lambda_{*}^{4}=\frac{4.69(1-a)}{\tau_{1}^{(\mathrm{Th})}\left[\left\langle B^{\prime 2}\right\rangle f_{B}+3 B_{0}^{2} \frac{\left\langle n^{\prime 2}\right\rangle}{n_{0}^{2}} f_{n} \cos ^{2} \Theta\right]}$.

For $\lambda \geq \lambda_{*}$ the positional angle tends to zero, when the wave electric field oscillations are perpendicular to the plane $(\boldsymbol{n} N)$. The maximum angle $\chi_{*}$ is related with $\lambda_{*}$ :

$\tan 2 \chi_{*}=0.4 \lambda_{*}^{2} B_{0} \frac{1-2 a}{1-a} \cos \Theta$.
The corresponding degree of polarization $p_{*}=p\left(\lambda_{*}\right)$ is:

$$
p_{*}=-9.14 \% \frac{1-\mu^{2}}{H(\mu)} \cdot \frac{1}{2 \sqrt{1+0.16 \lambda_{*}^{4} B_{0}^{2}\left(\frac{1-2 a}{1-a}\right)^{2} \cos ^{2} \Theta}} .
$$

Both these expressions and especially all the spectra can be used to estimate the characteristic turbulence parameters $R_{1},\left\langle B^{\prime 2}\right\rangle,\left\langle n^{\prime 2}\right\rangle$ in an atmosphere. This investigation is beyond the scope of this paper.

\section{Conclusion}

Let us summarize the results of the paper. First, the theory of radiative transfer in turbulent atmospheres is developed. For conservative atmospheres without regular and stochastic magnetic fields the new system of transfer equations for the average Stokes parameters $I_{0}, Q_{0}$ and $U_{0}$ formally coincides with the usual transfer equations if one introduces the effective optical depth instead of the Thomson one. It was demonstrated that the effective optical depth is smaller than the average optical depth. Thus, for semi-infinite conservative turbulent non-magnetized atmospheres the angular distribution of outgoing radiation and its polarization will be the same as for non-turbulent atmospheres. Turbulent atmospheres with absorption are described by transfer equations which differ from the usual equations. It is interesting to investigate what changes will appear for radiative transfer in such atmospheres.

Most prominently turbulent magnetized atmospheres change the picture of radiative transfer from that for nonturbulent magnetized atmospheres. In such atmospheres the fluctuations of the number density of free electrons and of the magnetic field give rise to an additional decrease of the magnitude of polarization and the position angle of the outgoing radiation. These effects change drastically the spectra of polarization degree and position angle. The spectrum $p(\lambda)$ acquires the form $\propto 1 / \lambda^{4}$ whereas atmospheres without magnetic field fluctuations give a $1 / \lambda^{2}$ dependence. The position angle $\chi(\lambda)$ tends to zero when the wave electric field oscillations are perpendicular to the plane $(\boldsymbol{n N})$ as in the case of non-magnetized atmospheres. New features of these spectra can be used to estimate the characteristic values of the atmospheric turbulence.

We obtained the asymptotic solution of the Milne problem for magnetized turbulent semi-infinite atmospheres. The simple analytical formulae for polarization degree and positional angle apply for sufficiently large Faraday rotation angles. Practically it is sufficient if at the unit Thomson optical depth there occurs only one revolution of the plane of polarization. The advantage of these analytical formulae is that the magnetic field direction can be arbitrary.

\section{References}

Agol, E., \& Blaes, O. 1996, MNRAS, 282, 965

Agol, E., Blaes, O., \& Ionescu-Zanetti, C. 1998, MNRAS, 293, 1

Basko, M. A. 1977, Afz, 12, 169

Chandrasekhar, S. 1950, Radiative Transfer (Oxford: Clarendon Press), 89 
Dolginov, A. Z., Gnedin, Yu. N., \& Silant'ev, N. A. 1995, Propagation and Polarization of Radiation in Cosmic Media (New York: Gordon \& Breach), 113

Gardiner, C. W. 1985, Handbook of Stochastic Methods (Berlin: Springer Verlag), 36

Gnedin, Yu. N., \& Silant'ev, N. A. 1997, Basic mechanisms of light polarization in cosmic media (Amsterdam: Hartwood Academic Publ.), 30

Kaminker, A. D., Pavlov, G. G., \& Shibanov, Yu. A. 1982, Ap\&SS, 86, 249

Meszaros, P., Nagel, W., \& Ventura, J. 1980, ApJ, 238, 1066

Nagel, W. 1980, ApJ, 236, 904
Shternin, P. S., Gnedin, Yu. N., \& Silant'ev, N. A. 2003, Astrophysics, 46,350

Silant'ev, N. A. 1979, SvA, 23, 21

Silant'ev, N. A. 1982, Ap\&SS, 82, 363

Silant'ev, N. A. 1994, JQSRT, 52, 207

Silant'ev, N. A. 2002, A\&A, 383, 326

Silant'ev, N. A. 2004, JETP, 98, 728

van Kampen, N. G. 1981, Stochastic Processes in Physics and Chemistry (Amsterdam: North Holland)

Varshalovich, D. A., Khersonsky, V. K., \& Moskalev, A. N. 1988, Quantum Theory of Angular Momentum (Singapore: World Scientific) 